\title{
Contactless Monitoring of the Diameter-Dependent Conductivity of GaAs Nanowires
}

\author{
Fauzia Jabeen ${ }^{1,2}$, Silvia Rubini ${ }^{2}(\bowtie)$, Faustino Martelli ${ }^{2}$, Alfonso Franciosi ${ }^{1,2}$, Andrei Kolmakov ${ }^{3}$, Luca Gregoratti ${ }^{1}$, \\ Matteo Amati ${ }^{1}$, Alexei Barinov ${ }^{1}$, Andrea Goldoni ${ }^{1}$, and Maya Kiskinova ${ }^{1}(\varangle)$ \\ ${ }^{1}$ Sincrotrone Trieste S. C. P. A., Elettra Laboratory, Area Science Park, S. S. 14, Km. 163.5, I-34149 Trieste, Italy \\ ${ }^{2}$ Laboratorio TASC IOM-CNR, Area Science Park, S. S. 14, Km. 163.5, I-34149 Trieste, Italy \\ ${ }^{3}$ Department of Physics, Southern Illinois University Carbondale, 1245 Lincoln Dr. Neckers 478, Carbondale, IL 62901-4401, USA \\ Received: 1 July 2010 / Revised: 12 August 2010 / Accepted: 12 August 2010 \\ C The Author(s) 2010. This article is published with open access at Springerlink.com
}

\begin{abstract}
Contactless monitoring with photoelectron microspectroscopy of the surface potential along individual nanostructures, created by the X-ray nanoprobe, opens exciting possibilities to examine quantitatively size- and surface-chemistry-effects on the electrical transport of semiconductor nanowires (NWs). Implementing this novel approach — which combines surface chemical microanalysis with conductivity measurements-we explored the dependence of the electrical properties of undoped GaAs NWs on the NW width, temperature and surface chemistry. By following the evolution of the Ga $3 \mathrm{~d}$ and As $3 \mathrm{~d}$ core level spectra, we measured the positiondependent surface potential along the GaAs NWs as a function of NW diameter, decreasing from 120 to $\sim 20 \mathrm{~nm}$, and correlated the observed decrease of the conductivity with the monotonic reduction in the NW diameter from 120 to $\sim 20 \mathrm{~nm}$. Exposure of the GaAs NWs to oxygen ambient leads to a decrease in their conductivity by up to a factor of 10, attributed to the significant decrease in the carrier density associated with the formation of an oxide shell.
\end{abstract}

\section{KEYWORDS}

Semiconductor nanowires, charge transport, surface oxidation, photoelectron X-ray microscopy, charging, GaAs

\section{Introduction}

Semiconductor nanowires (NWs) have unique and tunable physical and chemical properties and are promising building blocks for nanoelectronics, solar and fuel cells, chemical sensors, and biosensors [1-3]. The conductivity of NWs, one of the most important properties exploited in numerous applications, becomes extremely sensitive to the status of the surface with decreasing NW width, and dramatic conductivity changes can be observed when the Debye length becomes comparable to the NW radius [4]. Along with presence of adsorbates and charged surface states $[5,6]$, other factors that may affect the conductivity include the (i) reduced mobility due to enhanced phonon or surface scattering $[7,8]$, (ii) edge effects due to unsaturated bonds of the surface atoms [9], (iii) size-imposed limits to the effective doping concentration [9, 10], (iv) size-dependence of depletion width [11], band-gaps [12,13], and recombination barriers [14]. As a result, significant scatter in the conductivity data are observed for individual GaAs NWs

Address correspondence to Maya Kiskinova, kiskinova@elettra.trieste.it; Sivia Rubini, rubini@tasc.infm.it 
purportedly fabricated in the same manner [15].

Furthermore, the conductivity of undoped or lowdoped GaAs and other semiconductor NWs-that is expected to undergo substantial changes with decreasing width and in the presence of surface charges on the side walls [16] — can barely be characterized by conventional transport measurements. Chemical modifications of the surface are another poorly controlled factor that influences the transport properties, since these differ for NWs made of different compound semiconductors $[6,17]$. Formation of a thin oxide layer is the most common result of exposure to air $[6,18]$ and can lead to band bending and, consequently, to the formation of a depletion layer [19, 20].

An important prerequisite for understanding the impact of size and surface chemistry effects on the electrical properties is to be able to monitor simultaneously the changes in the NW diameter, surface composition and conductance. In this respect, X-ray photoelectron spectroscopy (XPS) has provided important contributions to semiconductor research, monitoring changes in the chemical state, band bending, and photoemission-induced surface charging $[12,18,19,21]$. Synchrotron light XPS has become a true microscopy technique, used recently for characterization of nanotubes, nanobelts, and NWs [22-24]. This study of undoped GaAs NWs prior to, and after, exposure to oxygen ambient demonstrates that by means of scanning photoelectron microscopy (SPEM) [25] it is possible to examine the interplay between NW diameter and conductance, combining full control of the surface chemical state with contactless monitoring of the changes in the conductivity along the axis of the NWs.

\section{Experimental}

The XPS microscopy experiments were carried out with the SPEM at the spectroscopy for chemical analysis (ESCA) microscopy beamline at the Elettra synchrotron facility in Trieste. In SPEM the incident photon beam is focused onto the sample to a spot of diameter, $D$, smaller than $0.15 \mu \mathrm{m}$ using zone plate optics. The SPEM has two operation modes, $\mu$-spot spectroscopy and imaging spectromicroscopy. The $\mu$-spot mode is identical to conventional XPS spectroscopy, i.e. energy distribution curves are measured from the selected area illuminated by the focused beam. The imaging mode maps the lateral distribution of elements by collecting photoelectrons with a selected energy while scanning the specimen with respect to the focused beam. More details about the instrument can be found at http://www.elettra.trieste.it/experiments/ beamlines/esca/index.html.

Using XPS to monitor the conductance changes in low-conducting nanostructures is in principle straightforward, since the ineffective screening of the electron holes created by photoemission results in the buildup of a positive surface potential, $V_{\mathrm{S}}$ [26-28], restricted within a relatively thin layer of $\leqslant 10 \mathrm{~nm}$, which is determined by the escape depth of the emitted electrons [29]. $V_{\mathrm{S}}$ grows until the photoelectron current $I_{\mathrm{ph}}=$ $\delta \cdot q \cdot F_{\mathrm{ph}} \cdot S$ becomes equal to the Ohmic neutralization current flowing between the irradiated spot and ground: i.e. $I_{\sigma}=\sigma \cdot V_{\mathrm{S}} \cdot S^{*} / x$, where $\delta$ is the electron yield, $q$ is the electron charge, $F_{\mathrm{ph}}$ is the photon flux, $S$ is the irradiated area, $\sigma$ is the NW conductivity, $S^{*}=\pi d_{x}^{2} / 4$ and $x$ are the effective NW cross-section and the conducting path length (i.e. the distance between the irradiated spot and the conductive and grounded substrate), respectively. At steady state, the value of $V_{\mathrm{S}}$ due to uncompensated charges is given by:

$$
V_{\mathrm{S}}=4 \cdot \delta \cdot q \cdot F_{\mathrm{ph}} \cdot S \cdot x / \sigma \cdot \pi d_{x}^{2}
$$

As a result, all photoelectrons emitted from the probed area undergo a rigid kinetic energy shift relative to the ground potential, $\Delta E_{\mathrm{K}}=V_{\mathrm{S}}$. Thus, in addition to chemical information, the sample conductivity can be inferred from the value of $\Delta E_{\mathrm{K}}$ measured from the XPS spectra.

GaAs NWs were grown by molecular beam epitaxy (MBE) on $n$-type GaAs (111)B deoxidized substrates. A $0.02 \mathrm{~nm}$ thick Au film, deposited in ultrahigh vacuum (UHV) was used as the growth catalyst [30]. NWs were oriented along the [111] substrate direction, with an average density of $1 \mathrm{NW} / \mu \mathrm{m}^{2}$. More details about the NW growth are given in Ref. [30]. To protect the surface of the NWs from oxidation, a thick amorphous As cap was deposited in the MBE chamber after the $\mathrm{NW}$ growth, and removed by heating at $450{ }^{\circ} \mathrm{C}$ after transfer of the sample into the UHV SPEM station. 
Photoemission analysis after decapping showed only the Ga and As spectra characteristic of atomically clean GaAs. Analysis with a scanning electron microscope (SEM) prior to, and after decapping, showed that the NW morphology and density remained unaffected by the capping/decapping cycle. The Ga and As core level binding energies, $E_{\mathrm{B}}$, are referred to the spectrometer Fermi level, determined from the $\mathrm{Au} 4 \mathrm{f}_{7 / 2}$ and $5 \mathrm{~d}$ spectra of a standard Au sample.

We investigated 6-10 $\mu \mathrm{m}$ long pencil-like NWs from two types of samples, A and B, fabricated in two different growth runs separated in time. The only difference between samples A and B was the nonintentional low doping, due to background impurities present in the MBE chamber, as measured by the Hall effect on GaAs calibration epilayers, grown just before the NW samples: $n$-type $\left(1.5 \times 10^{15} \mathrm{~cm}^{-3}\right)$ for A, and $p$-type $\left(8.1 \times 10^{15} \mathrm{~cm}^{-3}\right)$ for $\mathrm{B}$. As illustrated in Fig. 1(a), the NWs consist of a cylindrical base (a few $\mu \mathrm{m}$-long with a diameter of about $120 \mathrm{~nm}$ ) followed by a region of monotonically decreasing width (to 5-10 nm at the NW tip). The advantage of such NW morphology is that the width effects are probed on the same nanowire with improved statistics by measuring different NWs from the same sample grown under identical conditions. This eliminates uncertainties introduced by other factors when comparing NWs with different widths coming from different samples.

The low spatial density of the NWs allowed us to collect spectra from different spots along individual NWs, selected from the Ga 3d (or As 3d) SPEM images of the NW array in a cross-sectional geometry, as illustrated in Fig. 1(b). A schematic view of the contactless set-up used to monitor $V_{\mathrm{S}}$ by collecting spectra from different spots is sketched in Fig. 1(c). Since the electron binding energy, $E_{\mathrm{B}}$, the photon energy, $E_{\mathrm{ph}}$ and the kinetic energy of emitted electrons, $E_{\mathrm{K}}$, measured by the spectrometer work function, $\Phi$, are related through the equation: $E_{\mathrm{B}}=E_{\mathrm{ph}}-E_{\mathrm{K}}-\Phi$, in the following discussion we will refer solely to the changes in $E_{\mathrm{K}}, \Delta E_{\mathrm{K}}$, along the wire axis measured using focused beam with diameter $D=120 \mathrm{~nm}$, equal to or larger than the diameter of the NWs, $d$, as illustrated in Figs. 1(b) and $1(\mathrm{c})$.

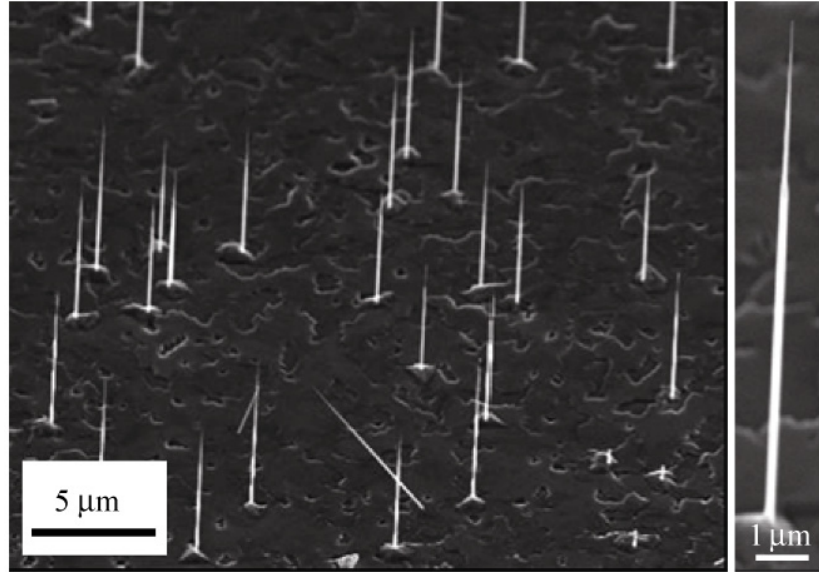

(a)

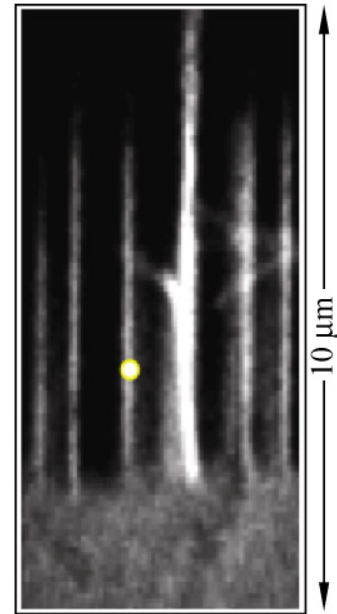

(b)

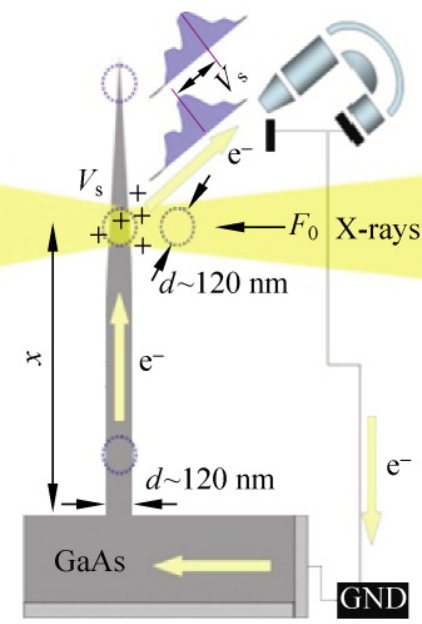

(c)
Figure 1 (a) SEM images of an ensemble of pencil-like NWs and of a single NW. (b) Ga 3d SPEM image of a NW array in crosssection used for selecting individual NWs for analysis. The yellow spot indicates the photon microprobe. (c) Schematic view of the contactless set-up to monitor the evolution of $V_{\mathrm{S}}$ by collecting spectra from different spots along the NW

\section{Results and discussion}

\subsection{Size-dependent conductivity of GaAs NWs and temperature effects}

The As 3d spectra in Fig. 2(a), measured at spots along the NW from the base towards the tip, clearly show that they undergo energy shifts, $\Delta E_{\mathrm{K}}$ with increasing distance, $x$, from the substrate. The Ga $3 \mathrm{~d}$ spectra showed very similar evolution, as evidenced by the plots of $\Delta E_{\mathrm{K}}$ vs. $x$ for As $3 \mathrm{~d}$ and Ga 3d in Fig. 2(b), measured for the $A$ and $B$ samples. The shifts are almost 
identical for the $\mathrm{A}$ and $\mathrm{B}$ samples, i.e. $\Delta E_{\mathrm{K}}$ reaches $-0.4 \pm 0.05 \mathrm{eV}$ when $d$ drops to $\sim 40 \mathrm{~nm}$. Moving further toward the tip, where $d$ becomes $20-25 \mathrm{~nm}$, the value of $\Delta E_{\mathrm{K}}$ rises to $-0.8 \pm 0.1 \mathrm{eV}$ (as shown in Fig. 2(c)). The larger spread of the shift for $d<40 \mathrm{~nm}$ is due to the lower SPEM accuracy in locating the spot and the decrease of the XPS signal. Spectra recorded moving further closer to the tip, where the NW diameter becomes comparable to the escape depth, are not considered.

The variations in $E_{\mathrm{K}}$ along the wire axis can, in principle, be attributed to any of the following three mechanisms: (i) changes in the chemical state of the surface; (ii) band bending (BB) and related surface photovoltage (SPV) effects, and (iii) build-up of positive $V_{\mathrm{S}}$ (charging), due to low sample conductivity. Our results rule out any major influence of the first two mechanisms. First, the XPS spectra do not show any variation in chemical composition along the NWs. Second, the values of $\Delta E_{\mathrm{K}}$ are the same for samples grown in different runs. Third, the widths of $\mathrm{As} 3 \mathrm{~d}$ and Ga $3 \mathrm{~d}$ spectra increase by $\sim 0.1 \mathrm{eV}$ with decreasing $d$. The widths of the spectra are not sensitive to BB and SPV effects, whereas charging can lead to peak broadening if the relaxation time for screening exceeds $\sim 10^{-7} \mathrm{~s}$, [26-28]; this indicates that the positiondependence of $E_{\mathrm{K}}$ is a consequence of charging. The considerably smaller value of $\Delta E_{\mathrm{K}}$ at $225^{\circ} \mathrm{C}$ reflects the increased NW conductivity (see Fig. 2(b)), which also supports the hypothesis that the origin of $\Delta E_{\mathrm{K}}$ is charging-related. In addition, the value of $\Delta E_{\mathrm{K}}$ in the same location varies when changing the photon flux, which is also clear evidence for charging effects. That is why all measurements were carried out with constant flux, controlled by the entrance slits.

The experimental value of $\Delta E_{\mathrm{K}}$ can be analyzed using a simple model for a semiconductor resistor with a steady-state potential of the irradiated spot at a distance $x$ from the NW base, $V_{\mathrm{S}}$. Since the estimated Debye length $L_{\mathrm{D}}=\left(\frac{\varepsilon \varepsilon_{0} k T}{e^{2} n_{0}}\right)^{1 / 2}$ for our low-doped GaAs

NWs is $130 \mathrm{~nm}$, which exceeds the maximum NW width $\left(d_{0}=120 \mathrm{~nm}\right)$, the NWs can be considered as electronically homogeneous in the radial direction. The value of $V_{\mathrm{s}}$ calculated according to the relationship (1)

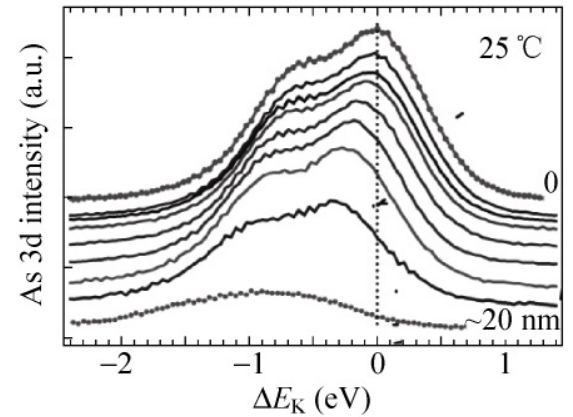

(a)

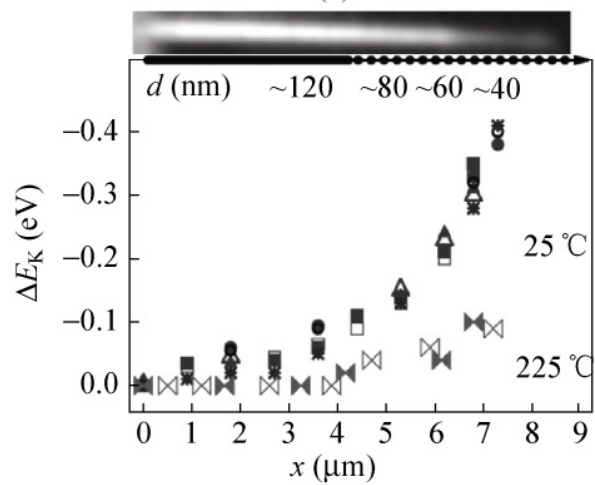

(b)

$d(\mathrm{~nm})$

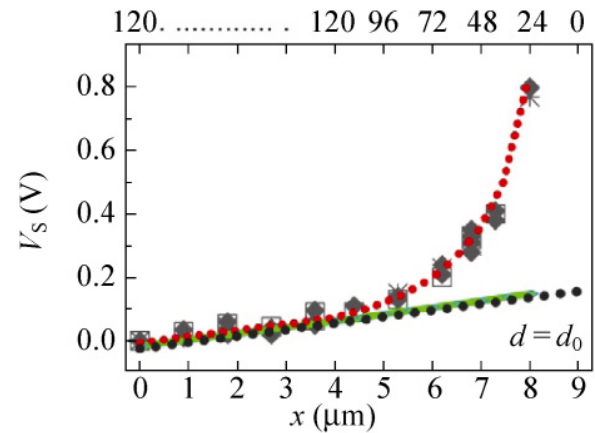

(c)

Figure 2 (a) As $3 \mathrm{~d}$ spectra recorded at different positions along the NW from the base toward the tip. The spectrum at point 0 , close to the substrate, is taken as the reference zero for $E_{\mathrm{K}}$. (b) Plots of the shift in $E_{\mathrm{K}}\left(\Delta E_{\mathrm{K}}\right)$ vs. the distance $x$ from the substrate (relative to ' 0 ') constructed using four sets of Ga $3 \mathrm{~d}$ (open symbols) and As $3 \mathrm{~d}$ spectra (solid symbols) measured from regions with $d \geqslant 40 \mathrm{~nm}$ on NWs from samples A and B at room temperature, and from sample B NWs at $225^{\circ} \mathrm{C}$. A representative SPEM Ga $3 \mathrm{~d}$ image is shown above the plot panel on the same scale of the $x$ axis. The NW diameter, $d$, as a function of $x$, as determined by higher resolution SEM images, is reported on the top axis of panel (c). (c) Calculated $V_{\mathrm{S}}$ values as a function of $x$ using Eq. (1) and assuming Ohmic behavior with constant $\sigma_{0}=0.15 \mathrm{~S} / \mathrm{m}$ : the dotted black line refers to a cylindrical NW with diameter $d_{0}$ and the green line to a pencil-like NW. The dotted red line that fits well with the experimental data is for pencil-like NWs assuming a linear decrease of $\sigma$ with decreasing $d$. The symbols denote experimental values of $\Delta E_{\mathrm{k}}$ for $\mathrm{Ga} 3 \mathrm{~d}$ (squares) and $\mathrm{As} 3 \mathrm{~d}$ (diamonds). The diameter, $d$, of the pencil-like NW is estimated for a $9 \mu \mathrm{m}$ long $\mathrm{NW}$ with a $4 \mu \mathrm{m}$ cylindrical part 
using $F_{\mathrm{ph}}=10^{11} \mathrm{ph} / \mu \mathrm{m}^{2}$ and $\delta=0.1$, reproduces well the experimental value of $\Delta E_{\mathrm{k}}$ for the cylindrical part with $d_{0}=120 \mathrm{~nm}$ and $0<x \leqslant L_{0}=4 \mu \mathrm{m}$ for NW conductivity with $\sigma_{0}=0.15 \mathrm{~S} / \mathrm{m}$ (shown by the red dotted line in Fig. 2(c)). This value is much lower than the values $\sigma=140 \mathrm{~S} / \mathrm{m}$ and $\sigma=33 \mathrm{~S} / \mathrm{m}$ of the GaAs epitaxial layers corresponding to samples A and B, respectively. Possible reasons for this may be: (i) the impurity incorporation during growth of the NWs is different from that in standard epitaxial growth, and/or (ii) the electron and hole relaxation times for the NWs are much lower than for bulk GaAs due to surface scattering or the presence of structural defects. Assuming Ohmic conductance, i.e. using the value $\sigma_{0}=0.15 \mathrm{~S} / \mathrm{m}$ evaluated for the cylindrical part, and approximating the pencil-like part as segments with stepwise decreasing diameter, we obtained a rather flat plot of $V_{\mathrm{S}}$ vs. $x$ (the blue line in Fig. 2(c)) for the conical part, that does not correspond to the rather rapid increase in $V_{\mathrm{S}}$ observed experimentally. This clearly indicates sizedependent conductivity. In fact, the electrical characteristics of our NWs resemble those of field-effect transistors, where the carrier concentration depends on changes in the charge at the surface. Because of the low density of charge carriers, the screening in semiconductors involves large length scales and, as the NW width decreases, the amount of the depleted zone as a fraction of the total volume increases. This means that moving towards the thinner part, the electron depleted zone on the sidewall of the NWs becomes increasingly important, i.e. to fit the experimental data, we have to use effective cross-sectional electron densities smaller than those predicted from the real NW widths. Assuming a linear decrease of the conductivity in the cone part $\sigma_{\mathrm{x}}=\sigma_{0}\left(1-c\left(x-L_{0}\right)\right)$, where $\sigma_{0}$ is $0.15 \mathrm{~S} / \mathrm{m}$, determined for the cylindrical part with $L_{0}=4 \mu \mathrm{m}$, we obtained a rather good fit for $c=0.2$ (the green line in Fig. 2(c)); this suggests a five-fold drop in the conductivity on reducing the diameter from 120 to $\sim 20 \mathrm{~nm}$. The suggested [8] enhanced role of the electron surface scattering and surface transport in increasing the surface to volume ratio-which scales linearly with $d$-are among the important factors that may account for the observed linear decrease of the NW conductivity. This also is in accordance with the dramatic effect of surface oxidation on the conductivity, reported in the next section. However, some contribution to the conductivity from inhomogeneous incorporation of impurities, due to changes in the NW growth mode, cannot be completely excluded [10].

\subsection{Oxidation effects on the conductivity of GaAs NWs}

When the GaAs NWs were exposed to oxygen, a shell of surface oxides was formed, as evidenced by the As 3d (not shown) and Ga 3d spectra (Fig. 3(a)). The correlation between the value of $\Delta E_{\mathrm{K}}$ and conductance in this case is not straightforward, since we should consider the contribution of chemical shifts and BB away from the interface, with the characteristic screening lengths. Formation of Ga oxides results in two components on the low kinetic energy side of the GaAsrelated Ga 3d peak, shifted by -0.7 and $-1.4 \mathrm{eV}$, whereas the multiple oxidation states of As result in up to five new components with shifts ranging between -0.7 and $-4.5 \mathrm{eV}[31,32]$. The As oxides may also undergo partial photon-induced reduction, which complicates the interpretation of the As 3d spectra [29, 33]. The smaller chemical shifts, fewer oxide components and higher stability of Ga oxides makes the Ga 3d spectra more suitable for exploring charging effects in the oxidized NWs.

The Ga 3d spectra in Fig. 3(a) contain both GaAsand Ga oxides-related components. Moving towards the NW tip all spectra shift to lower $E_{\mathrm{K}}$ accompanied by visible broadening. When the NW diameter drops below $\sim 80 \mathrm{~nm}$ (spectra $4-6$ ), the broadening and $E_{\mathrm{K}}$ become time-dependent, i.e. the appearance of the spectrum varies from scan to scan. The most likely mechanisms responsible for the observed spectral broadening and time-dependence are charging-occurring on a time scale comparable with the acquisition timeand differential charging resulting from dependence of $E_{\mathrm{K}}$ on the take-off angle [26, 27, 34]. The latter is a natural result of the geometry of the NWs since, with decreasing diameter, photoelectrons with variable takeoff angles start to contribute almost equally to the signal. Deconvolution of the Ga 3d spectra in Fig. 3 was carried out only for those with a steady-state surface potential, which allowed us to apply proportional broadening of the components resulting from 
charging. Comparison of the relative mass of the Ga oxide and $\mathrm{Ga}$ (GaAs) components indicates that the thickness of the oxide shell formed is of the order of $1 \mathrm{~nm}$. The conductance of the NWs can be significantly modified by the acceptor levels induced by the oxide shell. These can trap free carriers and create a depletion space-charge region extending deep inside the wires.

To gauge the degree of BB caused by the GaAsoxide interface, we compare the values of $E_{\mathrm{K}}$ of GaAsrelated Ga 3d spectra measured at the base of the NWs before and after oxidation. The observed difference in $E_{\mathrm{K}}$ was $-0.4 \mathrm{eV}$; this may also include some charging contribution. This value is much smaller than the significant shifts in $E_{\mathrm{K}}$ observed with decreasing NW width (Fig. 3), accompanied by broadening of both GaAsand oxide-related components. The As $3 \mathrm{~d}$ spectra showed the same trends in both $E_{\mathrm{K}}$ and lineshape. Similar values of $\Delta E_{K}$ were also measured for the valence band spectra onset, which reflects only the $\mathrm{BB}$ and charging effects.

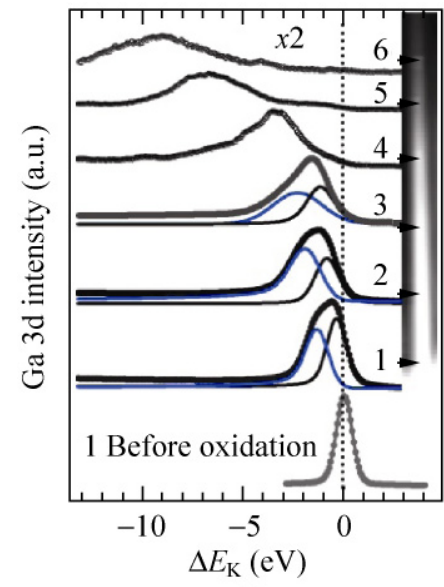

(a)

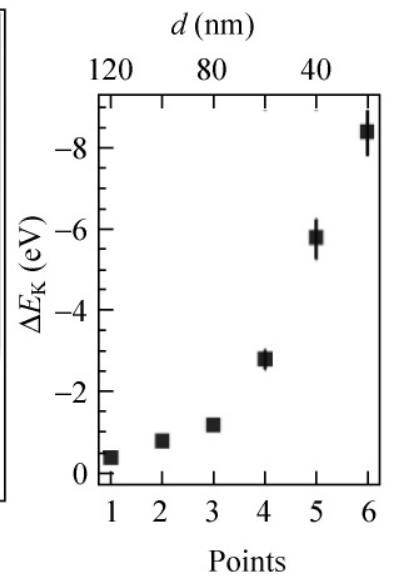

(b)
Figure 3 (a) Ga $3 d$ spectra measured at the positions along the NW indicated in the SPEM image on the right, after exposure to $1 \times 10^{-6} \mathrm{mbar}_{2}$ for $12 \mathrm{~h}$. The bottom spectrum is taken before oxidation at position 1. The deconvolution (GaAs-related (black) and oxide-related (blue) components) is shown for positions 1 to 3 . The spectra recorded at positions 4 to 6 are affected by the variation in $V_{\mathrm{S}}$ on a time scale comparable to the spectra acquisition time (see text). (b) Plot of $\Delta E_{\mathrm{K}}$ vs. $x$. The values of $\Delta E_{\mathrm{K}}$ correspond to the shifts in the maximum of the non-deconvoluted spectra with respect to the maximum in position 1 before oxidation. The vertical error bars account for the observed variations in the positions of the spectral maxima due to time-dependent charging
The Ga 3d spectra from spots (1-3) in Fig. 3 were stable during acquisition time and showed that a decrease of the NWs width from $\sim 120$ to $80 \mathrm{~nm}$ resulted in a value of $V_{\mathrm{S}} \sim 1 \mathrm{eV}$, compared to $\sim 0.2 \mathrm{eV}$ for pristine NWs (Fig. 2(a)). This corresponds to a drop in the conductivity by a factor of five, indicating a strong depletion of the carrier density in oxidized NWs. The temporal variations in the maxima of spectra 4-6, induced by time-dependent charging, were up to $\pm 0.5 \mathrm{eV}$. The values are much smaller than the values of $\Delta E_{\mathrm{K}}$, which exceed $8 \mathrm{eV}$, suggesting that on oxidation the NW regions closer to the tip have become semiinsulating. As noted above, in addition to the drastic reduction in the carrier density, increased carrier scattering by surface states may also contribute to such dramatic decreases in the conductivity.

\section{Conclusions}

We have demonstrated that the influence of size, temperature and surface treatment on the conductance of individual low-doped GaAs NWs can be addressed and quantified by contactless measurements of the photon beam-induced surface potential along a NW axis using photoelectron microspectroscopy. Estimates of the conductivity of low-conductive NWs-that cannot be reliably obtained by conventional transport measurements-are thus possible. The nearly linear decrease of the measured conductivity when continuously reducing the NWs diameter from 120 to $\sim 20 \mathrm{~nm}$, is direct evidence for the presence of size effects; this relevant to the design of devices using NWs with very small diameters. The dramatic drop in the conductivity of the NWs as result of the formation of an oxide shell provides direct evidence for the extreme sensitivity of the transport properties of these nanostructures to the ambient conditions, and the need for finding reliable surface passivation schemes. These results demonstrate the potential of this method for future investigations which decouple the impact of nanowire diameter, surface chemical state, metal contacts and gate voltage on conductivity. The resulting information will be vital if these nanomaterials are to be incorporated into various device structures as the active elements. 


\section{Acknowledgements}

This project was funded by Friuli Venezia Giulia L.R. 47/78-1953 Ambient and Biological Sensors. Participation of AK was supported through National Science Foundation (NSF) No. ECCS-0925837 grant. We thank Majid Kazemian Abyaneh for critical reading of the manuscript.

Open Access: This article is distributed under the terms of the Creative Commons Attribution Noncommercial License which permits any noncommercial use, distribution, and reproduction in any medium, provided the original author(s) and source are credited.

\section{References}

[1] Hayden, O.; Agarwal, R.; Lu, W. Semiconductor nanowire devices. Nanotoday 2008, 3, 12-22.

[2] Sun, Y.; Rogers, J. A. Inorganic semiconductors for flexible electronics. Adv. Mat. 2007, 19, 1897-1916.

[3] Lu, W.; Lieber, C. M. Semiconductor nanowires. J. Phys. D: Appl. Phys. 2006, 39, R387-R406.

[4] Dimitriev, S.; Lilach, Y.; Button, B.; Moskovits, M.; Kolmakov, A. Nanoengineered chemiresistors: The interplay between electron transport and chemisorption properties of morphologically encoded $\mathrm{SnO}_{2}$ nanowires. Nanotechnology 2007, 18, 055707.

[5] Zhang, S.; Hemesath, E. R.; Perea, D. E.; Wijaya, E.; Lensch-Falk, J. L.; Lauhon, L. J. Relative influence of surface states and bulk impurities on the electrical properties of $\mathrm{Ge}$ nanowires. Nano Lett. 2009, 9, 3268-3274.

[6] Wang, D.; Chang, Y. L.; Wang, Q.; Cao, J.; Farmer, D. B.; Gordon, R. G.; Dai, H. Surface chemistry and electrical properties of germanium nanowires. J. Am. Chem. Soc. 2004, 126, 11602-11611.

[7] Motayed, A.; Vaudin, M.; Davydov, A. V.; Melngailis, J.; He, M.; Mohammad, S. N. Diameter dependent transport properties of gallium nitride nanowire field effect transistors. Appl. Phys. Lett. 2007, 90, 043104.

[8] Ford, A. C.; Ho, L. C.; Chueh, Y. L.; Tseng, Y. C.; Fan, Z.; Guo, J.; Bokor, J.; Javey, A. Diameter-dependent electron mobility of InAs nanowires. Nano Lett. 2009, 9, 360-365.

[9] Khanal, D. R.; Yim, J. W. L.; Walukiewicz, W.; Wu, J. Effects of quantum confinement on the doping limit of semiconductor nanowires. Nano Lett. 2008, 7, 1186-1190.

[10] Perea, D. A.; Hernesath, E. R.; Schwalbach, E. J.; Lensch- Falk, J. L.; Voorhees, P. W.; Lauhon, L. J. Direct measurement of dopant distribution in an individual vapour-liquid-solid nanowire. Nat. Nanotechnol. 2009, 4, 315-319.

[11] Simpkins, B. S.; Mastro, M. A.; Eddy, C. R.; Pehrsson, P. E. Surface depletion effects in semiconducting nanowires. $J$. Appl. Phys. 2008, 103, 104313.

[12] Dong, A.; Yu, H.; Wang, F.; Buhro, W. E. Colloidal GaAs quantum wires: Solution-liquid-solid synthesis and quantumconfinement studies. J. Am. Chem. Soc. 2008, 130, 59545961.

[13] Pan, H.; Feng, Y. P. Semiconductor nanowires and nanotubes: Effects of size and surface-to-volume ratio. ACS Nano 2008, 2, 2410-2414.

[14] Calarco, R.; Marso, M.; Richter, T.; Aykanat, A. I.; Meijers, R.; Hart, A. V. D.; Stoica, T.; Lüth, H. Size-dependent photoconductivity in MBE-grown $\mathrm{GaN}$ nanowires. Nano Lett. 2005, 5, 981-984.

[15] Schricker, A. D.; Davidson, F. M.; Wiacek, R. J.; Korgel, B. A.; Space charge limited currents and trap concentrations in GaAs nanowires. Nanotechnology 2006, 17, 2681-2688.

[16] Elfstrom, N.; Juhasz, R.; Sychogov, I.; Engfeldt, T.; Karlstrom, A. E.; Linnros, J. Surface charge sensitivity of silicon nanowires: Size dependence. Nano Lett. 2007, 7, 2608-2612.

[17] Chen, H. Y.; Chen, R. S.; Chang, F. C.; Chen, L. C.; Chen, K. H.; Yang, Y. J. Size-dependent photoconductivity and dark conductivity of $m$-axial GaN nanowires with small critical diameter. Appl. Phys. Lett. 2009, 95, 143123.

[18] Gu, W.; Choi, H.; Kim, K. Universal approach to accurate resistivity measurement for a single nanowire: Theory and application. Appl. Phys. Lett. 2006, 89, 253102.

[19] Yu, P. Y.; Cardona, M. Fundamentals of Semiconductors: Physics and Material Properties; Springer-Verlag: Berlin, 2001; pp 464.

[20] Schubert, E.; Razek, N.; Frost, F.; Schindler, A.; Rauschenbach, B. GaAs surface cleaning by low-energy hydrogen ion bombardment at moderate temperatures. J. Appl. Phys. 2005, 97, 023511.

[21] Margaritondo, G. Synchrotron light in semiconductor research: Three decades of revolution. J. Phys. IV 2006, 132, 23-29.

[22] Barinov, A.; Üstünel, H.; Fabris, S.; Gregoratti, L.; Aballe, L.; Dudin, P.; Baroni, S.; Kiskinova, M. Defect-controlled transport properties of metallic atoms along carbon nanotube surfaces. Phys. Rev. Lett. 2007, 99, 046803.

[23] Kolmakov, A.; Potluri, S.; Barinov, A.; Mentes , T. O.; Gregoratti, L.; Niño, M. A.; Locatelli, A.; Kiskinova, M. Spectromicroscopy for addressing the surface and electron transport properties of individual 1-D nanostructures and their networks. ACS Nano 2008, 2, 1993-2000.

[24] Barinov, A.; Gregoratti, L.; Dudin, P.; La Rosa, S.; Kiskinova, M. Imaging and spectroscopy of multiwalled carbon nanotubes 
during oxidation: Defects and oxygen bonding. Adv. Mater. 2009, 21, 1916-1920.

[25] Barinov, A.; Dudin, P.; Gregoratti, L.; Locatelli, A.; Menteş, T. O.; Niño, M. A.; Kiskinova, M. Synchrotron-based photoelectron microscopy. Nucl. Instr. Meth. Phys. Res. A 2009, 601, 195-202.

[26] Cazaux, J. Mechanisms of charging in electron spectroscopy. J. Electr. Spectr. Rel. Phenom. 1999, 105, 155-185.

[27] Cazaux, J. Secondary electron emission and fundamentals of charging mechanisms in XPS. J. Electr. Spectr. Rel. Phenom. 2010, 178-179, 357-372.

[28] Günther, S.; Kolmakov, A.; Kovac, J.; Kiskinova, M. Artefact formation in scanning photoelectron emission microscopy. Ultramicroscopy 1998, 75, 35-51.

[29] de Groot, F.; Kotani, F. A. Core Level Spectroscopy of Solids (Advances in Condensed Matter Science Vol. 6); Taylor and Francis: New York, 2008; pp 231.

[30] Piccin, M.; Bais, G.; Grillo, V.; Jabeen, F.; de Franceschi, S.;
Carlino, E.; Lazzarino, M.; Romanato, F.; Businaro, L.; Rubini, S.; Martelli, F.; Franciosi, A. Growth by molecular beam epitaxy and electrical characterization of GaAs nanowires. Physica E 2007, 37, 134-137.

[31] Hale, M. J.; Yi, S. I.; Sexton, J. Z.; Kummel, A. C.; Passlack, M. Scanning tunneling microscopy and spectroscopy of gallium oxide deposition and oxidation on $\operatorname{GaAs}(001)-c(2 \times 8) /(2 \times 4)$. J. Chem. Phys. 2003, 119, 6719-6728.

[32] Palomares, F. J.; Alonso, M.; Jimenez, I.; Avila, J.; Sacedon, J. L.; Soria, F. Electron beam induced reactions of O2/GaAs interface. Surf. Sci. 2001, 482-485, 121-127.

[33] Mori, G.; Lazzarino, M.; Ercolani, D.; Sorba, L.; Heun, S.; Locatelli, A. Desorption dynamics of oxide nanostructures fabricated by local anodic oxidation nanolithography. $J$. Appl. Phys. 2005, 97, 114324.

[34] Gonska, H.; Freund, H. J.; Hohlneicher, G. On the importance of photoconduction in ESCA experiments. J. Electr. Spec. Rel. Phenom. 1977, 12, 435-441. 